\title{
Effect of homogenization speed on the stability of the mayonnaise from catfish belly and red palm mixture oil
}

\author{
Dewi Fortuna Ayu ${ }^{1 *}$, Vonny Setiaries Johan ${ }^{1}$, Netti Herawai, and Kiki Amelia Napitupulu ${ }^{1}$ \\ ${ }^{1}$ Departement of Agricultural Technology, Riau University, Pekanbaru, 28293, Indonesia
}

\begin{abstract}
This study aimed to determine the effect of homogenization speed on the stability of mayonnaise from catfish belly and red palm mixture oil. This study used a non-factorial complete randomized design (CRD) with five treatments and three replications. The treatments were homogenization speed by using a hand mixer, namely: K1= scale $1(658$ $\mathrm{rpm}), \mathrm{K} 2=$ scale $2(728 \mathrm{rpm}), \mathrm{K} 3=$ scale $3(845 \mathrm{rpm}), \mathrm{K} 4=$ scale $4(936$ $\mathrm{rpm})$, and K5= scale $5(1.028 \mathrm{rpm})$. Data were analyzed statistically using analysis of variance and followed by Duncan's multiple range test (DMRT) at the $5 \%$ level. The results of the analysis showed that the homogenization speed significantly affected the stability of the mayonnaise on the viscosity, creaming index on the 10th day of storage, and stability of the emulsion by centrifugation on the 5th and 10th days of storage. The homogenization speed also affected the sensory descriptive of the mayonnaise on the viscosity. The best treatment was K5 (1.028 rpm) with $22.470 \mathrm{mPa}$.s viscosity, $100 \%$ creaming index on the 10 th day of storage, $100 \%$ emulsion stability on the 5 th day of storage, and $98,67 \%$ on the 10 th day of storage. The mayonnaise was very yellow in color, normal taste, and thick.
\end{abstract}

\section{Introduction}

Catfish (Pangasius sp.) is one of the main commodities of aquaculture in Riau Province. The production of catfish in Riau Province in 2019 was 27,335 tons. The highest production of catfish in Riau Province comes from Kampar Regency, which was 16,465 tons [1]. Rodiah et al. [2] stated that the people of Kampar Regency generally process catfish by smoking fish as salai fish. Fresh catfish are processed as 10-15 tons/day and produce into salai fish as much as 5-7 tons/day. The salai fish processing produced waste of around 3-5 tons/day and has not been utilized optimally.

Research has succeeded in extracting crude fish oil from the stomach contents of catfish and yielding about 20.34-30.05\% [3]. The fatty acids composition of the catfish oil waste were $33.95 \%$ palmitic acid, $35.85 \%$ oleic acid, and $12.35 \%$ polyunsaturated fatty

\footnotetext{
${ }^{*}$ Corresponding author: fortuna_ayu2004@yahoo.com
} 
acids (PUFA), in the form of linoleic, linolenic, eicosapentaenoic (EPA), and docosahexaenoic acid (DHA). The high PUFA content, especially EPA and DHA, allows fish oil to be susceptible to oxidation reactions that cause unwanted color, odor, and taste, and loss of nutritional value [4].

One of the strategies that can be used to prevent oil oxidation in emulsion systems is the addition of natural antioxidants. One of the vegetable oil rich in antioxidant components that can be added to fish oil is red palm oil (RPO). According to previous research, total carotene content in RPO ranged from $524-542 \mathrm{mg} / \mathrm{kg}$ and total tocols $875 \mathrm{mg} / \mathrm{kg}$, which consist of $242 \mathrm{mg} / \mathrm{kg} \alpha$-tocopherol, $266 \mathrm{mg} / \mathrm{kg} \alpha$-tocotrienol, and $367 \mathrm{mg} / \mathrm{kg}$ ocotrienol [5]. The research results of other research showed that the minor components ( $\beta$-carotene, tocopherol, and tocotrienols) in RPO had a synergistic antioxidant activity which contributed to the photo-oxidation stability of RPO during storage at high light intensity [6]. The utilization of RPO in addition to fish oil to improving the photo-oxidation stability was also expected to enrich the functional properties of the mixture oil.

Mayonnaise is an oil-in-water emulsion. An emulsion consists of a dispersion phase (internal phase), a dispersion medium (external phase), and an emulsifying agent. The emulsion formation required energy, such as mechanical force, to break up the liquid, thereby increasing the surface area of the internal phase [7]. Therefore, in the process of making mayonnaise, a homogenization process is needed as a mass mixing process. During the mixing process, the rotating speed of the mixer used will cause a shear force on the emulsion which allowed changes in the physical properties of the emulsion [8]. This research aim was to determine the effect of homogenization speed on the stability of mayonnaise from catfish belly and red palm mixture oil.

\section{Materials and methods}

\subsection{Materials and equipment}

The materials used for the extraction and purification of catfish oil were catfish belly fat, acetic acid, bentonite, and $\mathrm{NaOH}$. The ingredients for making mayonnaise consist of pure catfish oil, RPO, egg yolk, salt, sugar, mustard, lime juice, and water. Red palm oil is made from crude palm oil (CPO) which was purchased from PT. Salim Ivomas Pratama (Bimoli) and purified without bleaching process at the SEAFAST Laboratory of IPB Bogor. The catfish belly fat was taken from the waste of a small industry of smoked catfish at District XIII Koto Kampar, Kampar Regency. The equipment used in this study consisted of a Philips HR-1552 hand mixer, oven, filter container, dark glass bottle, stopwatch, $\mathrm{pH}$ meter, Brookfield viscometer, centrifuge, cups, and tools used for the sensory test.

\subsection{Research methods}

This research was carried out experimentally using a completely randomized design (CRD) consisting of 5 treatments. The treatment in this study consisted of variations in the rotational speed of the hand mixer, namely K1 = Scale $1(658 \mathrm{rpm}), \mathrm{K} 2=$ Scale $2(728$ $\mathrm{rpm}), \mathrm{K} 3=$ Scale $3(845 \mathrm{rpm}), \mathrm{K} 4=$ Scale $4(936 \mathrm{rpm})$, and K5 = Scale 5 (1,028 rpm). Each treatment was repeated three times. The mayonnaise formulation consists of $56 \mathrm{~g}$ catfish oil, $14 \mathrm{~g}$ red palm oil, $12 \mathrm{~g}$ chicken egg yolk, $9.84 \mathrm{~g}$ water, $5.6 \mathrm{~g}$ lemon juice, 0.96 g salt, $0.96 \mathrm{~g}$ sugar, and 0.64 mustard.

The stirring time in making mayonnaise for each sample was \pm 12 minutes. The initial stage of making mayonnaise was egg yolks mixed with water and dry ingredients such as salt, sugar, and mustard, then stirred using a hand mixer at a speed according to treatment 
for 2 minutes. The next step was to add the catfish belly oil and RPO little by little $( \pm 7$ minutes) to form an oil-in-water emulsion. After the emulsion formed, lemon juice was added little by little ( \pm 1 minute) and followed by the stirring process for 2 minutes and become the mayonnaise emulsion. The resulting mayonnaise was then put into a glass jar, tightly closed, and stored for 10 days at room temperature until analyzed.

\subsection{Observation}

Observation on the mayonnaise consists of viscosity, creaming index test, stability test by centrifugation, and organoleptic test on color, aroma, and appearance. The organoleptic test was carried out by presenting a mayonnaise sample of $5 \mathrm{~g}$ in a clean container and coded with random numbers. Each panelist was asked to assess the color, aroma, and appearance of the mayonnaise for the descriptive and hedonic attributes and write down the results of the assessment on the questionnaire sheet provided.

\subsection{Data analysis}

The data obtained were analyzed statistically using analysis of variance (ANOVA) in SPSS software version 16.0. If the F count was higher than the F table, it will be continued with Duncan's new multiple range test (DNMRT) for a further test at a 5\% level.

\section{Results and discussion}

\subsection{Viscosity}

Viscosity is a value that indicates the unit thickness of the dispersion medium in an emulsion system. The viscosity of a preparation describes the flow properties of the preparation. The viscosity average of the mayonnaise from catfish belly and red palm mixture oil can be seen in Fig. 1.

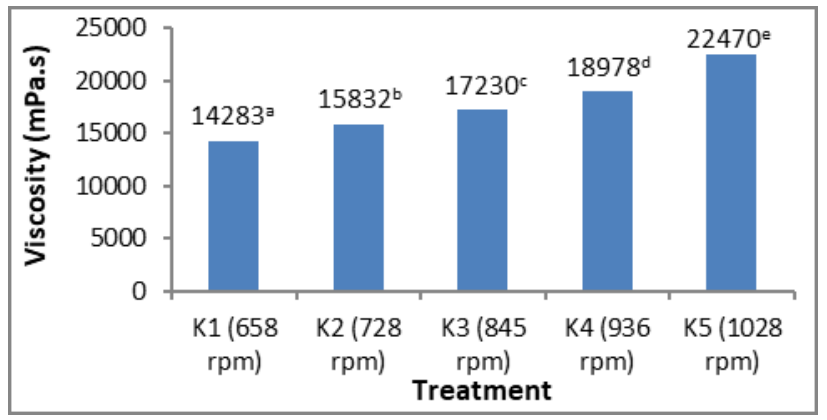

Fig. 1. The viscosity average of the mayonnaise from catfish belly dan red palm mixture oil

Fig.1. shows that the viscosity of the mayonnaise ranges from 14,283.67-22,470.00 $\mathrm{mPa}$.s. The highest viscosity was obtained in treatment $\mathrm{K} 5(1,028 \mathrm{rpm})$ which was $22,470.00 \mathrm{mPa}$.s and the lowest viscosity was obtained in treatment $\mathrm{K} 1(658 \mathrm{rpm})$ which was $14,283.00 \mathrm{mPa}$.s. The viscosity of the mayonnaise increased with the increase of the homogenization speed during the mayonnaise made processing. This was because the higher the homogenization speed, the greater kinetic energy was occurred to made the emulsifier more able to stabilize the droplets in the mayonnaise emulsion. 
The speed of homogenization affected the particle size distribution of the mayonnaise. The increasing speed of homogenization in the making process of the mayonnaise, the smaller particle size produced in the emulsion. Energy input has a direct effect on the mayonnaise particle size. The result was following the previous research which stated that the greater the increase in the given kinetic force, the smaller the particle size [9]. The smaller the droplet size formed, the higher the volume ratio which allows the bond between droplets to be stronger so that the film layer is stiffer and the viscosity increases [10].

\subsection{Creaming index}

Creaming index is a parameter that can indicate the instability of the emulsion because it is an event where the droplets collect into one layer and then separate from the outer phase. The creaming index value is obtained by observing and measuring the volume of creaming that occurs. The creaming index of mayonnaise from catfish belly dan red palm mixture oil was calculated on 0, 5, and 10 days of storage can be seen in Fig.2.

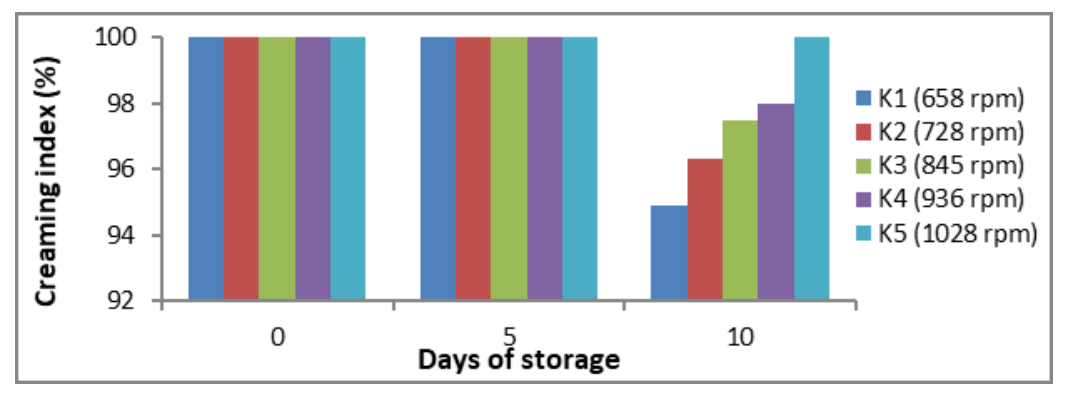

Fig. 2. The average creaming index of mayonnaise from catfish belly dan red palm mixture oil

Fiq.2. shows that the creaming index of mayonnaise on 0 and 5 days of storage in all treatments (K1, K2, K3, K4, and K5) was $100 \%$, which indicates that no separation of the oil, water, and creaming formed. The creaming index on the 10th day of observation ranged from $94.9-100 \%$. The creaming mayonnaise index on the 10th day of observation in $\mathrm{K} 1(658 \mathrm{rpm})$ was $94.9 \%$, not significantly different from the creaming mayonnaise index in K2 (96.3\%), K3 (97.5\%), and K4 (98.0\%). The creaming mayonnaise index value increases with the increasing speed of homogenization used. This shows that the higher the speed of homogenization used in the making of mayonnaise, the index value of creaming mayonnaise increases, and the index value of creaming mayonnaise decreases with increasing storage time. According to previous research, this was due to the greater speed of homogenization that will produce greater kinetic energy to make the emulsifier more able to stabilize the droplets in mayonnaise and produce smaller droplets so that the surface area of contact with the emulsifier is increasingly extensive [10]. As a result, the bond between droplets was stronger so that the film layer was more rigid and prevents the free movement of droplets from colliding with each other and joining to form larger droplets.

\subsection{Stability test by centrifugation}

Centrifugation is one method to test the stability of an emulsion. The centrifugation process is carried out to provide a shock to the emulsion preparation so that it can be seen how much damage occurs to the mayonnaise emulsion system. The stability of the mayonnaise emulsion from catfish belly and red palm mixture oil was observed on 0,5 , and 10 days of storage can be seen in Fig.3. 


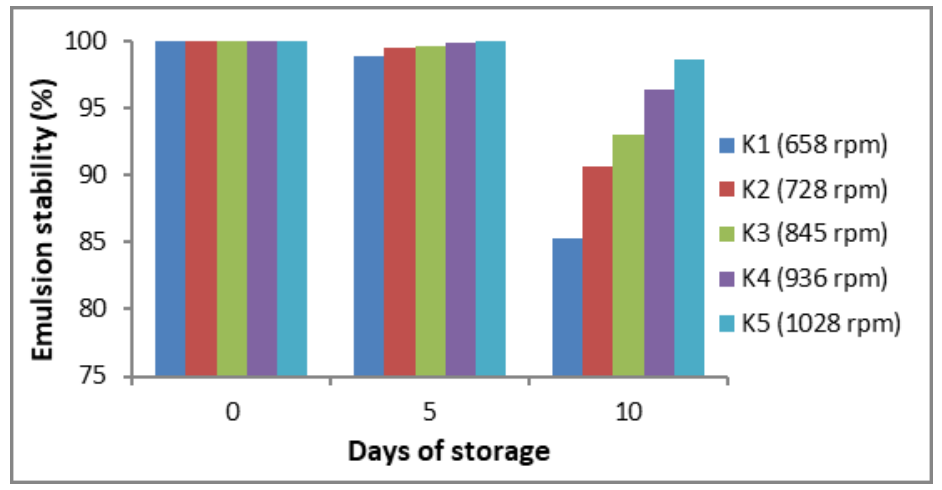

Fig. 3. Average stability of mayonnaise emulsion from catfish belly dan red palm mixture oil

Fig.3. shows the emulsion stability of treatment K1, K2, K3, K4, and K5 on 0, 5, and 10 days of storage. The higher the emulsion stability value, the better the level of emulsion stability. The stability of the mayonnaise emulsion in $\mathrm{K} 1, \mathrm{~K} 2, \mathrm{~K} 3, \mathrm{~K} 4$, and $\mathrm{K} 5$ on 0 days of storage was $100 \%$. The stability of the mayonnaise emulsion on day 5 ranged from 98.83 $100 \%$. The stability of the mayonnaise emulsion on the 5 th day of K5 treatment $(1,028$ $\mathrm{rpm}$ ) was $100 \%$ not significantly different from the stability of the mayonnaise emulsion in the treatment of K2 $(99.33 \%), \mathrm{K} 3(99.67 \%)$, and K4 $(99,83 \%)$. The stability of the mayonnaise emulsion on the 10th day of observation ranged from 85.33-98.67\%. The highest emulsion stability on the 10th day was obtained at treatment K5 $(1,028 \mathrm{rpm})$ which was $98.67 \%$ and the lowest emulsion stability was obtained at treatment K1 (658 rpm) which was $85.33 \%$. The stability of the mayonnaise emulsion on the 5th and 10th-day observations increased with the increase in the homogenization speed used. This indicated that the higher the homogenization speed used, the stability of the emulsion increases. The increase in emulsion stability in this study was not in line with the results of the previous study [11], namely the stability of the cream emulsion decreases along with the increasing speed of homogenization caused by the presence of internal and external factors that affect the stability of the emulsion.

\subsection{Organoleptic}

\subsubsection{Color}

Color is one of the important sensory parameters in assessing a food product. The color can give clues about chemical changes that occur in food. The average color, aroma, and thickness assessment descriptively and by hedonic mayonnaise is presented in Table 1 .

Table 1 shows that the results of a descriptive assessment of the color of the mayonnaise mixture of catfish belly oil and RPO ranged from 3.53-3.93 (very yellow in color) and the hedonic assessment ranged from 3.63 to 3.83 (like). The descriptive and hedonic assessment of mayonnaise color in each treatment was not significantly different. This was in line with the results of research [12], the variation of stirring speed in the formulation of gamma oryzanol nano cream does not affect the color of the resulting preparation. This was because the type and ratio of materials used in each treatment are the same. Also, the color of the mayonnaise produced was not much different from the color of commercial mayonnaise which is cream and golden yellow so that the panelists still can accept it. 
Table 1. Average sensory assessment of mayonnaise color from catfish belly dan red palm mixture oil

\begin{tabular}{|c|c|c|c|c|c|c|}
\hline \multirow{2}{*}{ Treatments } & \multicolumn{2}{|c|}{ Color score } & \multicolumn{2}{c|}{ Aroma score } & \multicolumn{2}{c|}{ Thickness score } \\
\cline { 2 - 7 } & Descriptive & Descriptive & Descriptive & Hedonic & Descriptive & Hedonic \\
\hline $\begin{array}{c}\text { K1= scale 1 } \\
(658 \text { rpm })\end{array}$ & 3.53 & 3.07 & 3.07 & 2.97 & $3.14^{\mathrm{a}}$ & 3.77 \\
\hline $\begin{array}{c}\text { K2= scale 2 } \\
(728 \text { rpm })\end{array}$ & 3.67 & 3.27 & 3.27 & 3.07 & $3.29^{\mathrm{ab}}$ & 3.60 \\
\hline $\begin{array}{c}\text { K3= scale 3 } \\
(845 \mathrm{rpm})\end{array}$ & 3.80 & 2.80 & 2.80 & 3.17 & $3.86^{\mathrm{bc}}$ & 3.83 \\
\hline $\begin{array}{c}\text { K4= scale 4 } \\
(936 \mathrm{rpm})\end{array}$ & 3.67 & 2.80 & 2.80 & 3.33 & $3.71^{\mathrm{bc}}$ & 3.87 \\
\hline $\begin{array}{c}\text { K5= scale 5 } \\
(1.028 \mathrm{rpm})\end{array}$ & 3.93 & 2.73 & 2.73 & 2.97 & $4.21^{\mathrm{c}}$ & 3.77 \\
\hline
\end{tabular}

Description: Descriptive color score: 1. white; 2. yellowish white; 3. yellow; 4. very yellow; 5. orange-yellow. Descriptive aroma score: 1 . strongly smells of RPO; 2 . smells of RPO; 3. normal; 4. smell of fish oil; 5 . strongly smells of fish oil. Descriptive thickness score: 1. very watery; 2 . watery; 3 . slightly watery; 4 . thick, 5 ; very thick. Hedonic score: 1. very dislike; 2 . dislike; 3 . kind alike; 4: like; 5: very like.

\subsubsection{Aroma}

Aroma is one of the quality parameters that determine the level of consumer acceptance of a food product. The aroma can describe the state of a product whether the product is suitable for consumption or not. Table 1 shows that the results of a descriptive assessment of the mayonnaise aroma from catfish belly and RPO mixture oil ranged from 2.73 to 3.27 (normal) and hedonic assessment ranged from 2.97 to 3.33 (somewhat like. The descriptive and hedonic assessment of the mayonnaise aroma attribute in each treatment was not significantly different. This was because the difference in the speed of homogenization used in the making of mayonnaise did not affect the aroma of the mayonnaise produced. These results were in line with previous research which indicated that the variation in the speed of homogenization in the making of nanoparticle cream did not affect the aroma of the cream produced, but changed in aroma when exposed to sunlight which accelerated fat oxidation [11]. According to other research, mayonnaise with the use of the same amount of oil as its composition does not affect the aroma of the mayonnaise produced [13]. The presence of volatile compounds derived from catfish belly oil and RPO was what makes the panelists less like (somewhat like) the aroma of the mayonnaise.

\subsubsection{Thickness}

Thickness is one of the texture attributes used to assess the physical properties and stability of the emulsion. Table 1 shows that the average descriptive thickness of the mayonnaise from catfish belly and RPO mixture oil ranged from 3.14-4.21 (slightly watery to thick). The mayonnaise viscosity score of K1 (3.14) was not significantly different from K2 (3.297). The mayonnaise viscosity score of K5 (4.21) was not significantly different from $\mathrm{K} 4$ (3.71) and K3 (3.86). This shows that the viscosity of mayonnaise increases with the increase in the speed of homogenization used in the making of mayonnaise. During the mixing process, the rotating speed of the mixer will cause a shearing force on the mayonnaise constituent particles to determine the size of the droplet size in the emulsion which allows changes in the physical properties of the emulsion [8]. The higher the speed 
of homogenization used, the smaller the droplet size, so the viscosity of the mayonnaise will increase as indicated by the thicker the mayonnaise produced.

The hedonic viscosity of the mayonnaise in each treatment was not significantly different, ranged from 3.60 to 3.87 (like). The resulting mayonnaise showed a good level of stability, which was indicated by the absence of separation between the water and oil phases. The viscosity of the mayonnaise was not much different from the viscosity of the commercial mayonnaise so that the panelists can accept it and give a favorable assessment of all mayonnaise treatments.

\section{Conclusion}

The homogenization speed treatment in making mayonnaise from catfish belly and RPO mixture oil significantly affected the stability of the mayonnaise on the viscosity, creaming index on the 10th day of storage, and the stability of the emulsion by centrifugation on the 5 th and 10th days of storage. The homogenization speed also affected the descriptive viscosity mayonnaise. The best treatment was K5 (1,028 rpm) with the viscosity of 22,470 mPa.s, $100 \%$ creaming index on the 10 th day of storage, $100 \%$ emulsion stability on the 5th day of storage, and $98.67 \%$ on the 10 th day of storage. The mayonnaise was very yellow in color, had normal flavor, and was thick.

\section{References}

1. BPS Riau. Riau dalam Angka 2019. Badan Pusat Statistik Provinsi Riau. Riau. (2021)

2. N.S. Rodiah, S.B.D. Bagus, B. Jamal, H. Ema, J. Penelitian dan Pengembangan Kelautan dan Perikanan. 11(2): 171-182. (2016)

3. E. Hastarini, D. Fardiaz, H.E. Irianto, S. Budhijanto, J. of Agritech. 32(4): 403-410. (2012)

4. J.C. Sullivan, S.M. Budge, S.T. Onge, J. Lipids. 46(2): 87-93. (2011)

5. J. Yie, M.L. Andersen, L.H. Skibsted, J.Food Chemistry. 127(3): 1792-1797. (2011)

6. D.F. Ayu, N. Andarwulan, P. Hariyadi, E.H. Purnomo, Food Sci. Biotechnol. 25: 401407. (2016)

7. M.E. Aulton. Pharmaceutical Practice. Longman Singapore Publishers Ptc Ltd. Singapore. (2002)

8. M.M. Amiji, B.J. Sandmann. Applied Physical Pharmacy. McGraw Hill Companies Inc, United States of America. (2003)

9. M.N. Fischer, D. Suppiger, E.J. Windhab, J. of Food Engineering. 80(1): 306-316. (2006)

10. H. Mollet, A. Grubeirmaen. (2001). Formulation Technology: Emulsion, Suspensions, Solid Forms. Wiley-vch Verlag.

11. G. Suprobo, R. Dwinna, J. Litbang Industri. 5(1): 1-12. (2015)

12. S. Dianmurdedi. Formulasi Nanokrim Gamma Oryzanol Menggunakan Metode Emulsifikasi Energi Tinggi dengan Variasi Kecepatan Pengadukan. Skripsi. Universitas Islam Negri Syarief Hidayatullah. Jakarta. (2018)

13. D.F. Ayu, T.S. Lumbangaol, A. Diharmi, J.Teknologi dan Industri Pertanian Indonesia. 12(2): 63-70. (2020) 